\title{
Pathological Narcissism in Adolescents: Relationships with Childhood Maltreatment and Internalizing and Externalizing Difficulties
}

Karin Ensink $^{1 *}$, Simon Chrétien ${ }^{1}$, Deanne Daigle ${ }^{1}$, Lina Normandin $^{1}$ Michaël Bégin $^{1} \&$ Peter Fonagy ${ }^{2}$

\author{
${ }^{1}$ Université Laval \\ École de psychologie \\ 2325 rue des Bibliothèques \\ Québec (QC), Canada G1V 0A6 \\ ${ }^{2}$ University College London \\ Psychoanalysis Unit \\ Research Department of Clinical, Educational and Health Psychology \\ Gower Street \\ London, UK WC1E 6BT
}

*Address correspondence to this author at:

Mailing: 1426 Pavillion Félix-Antoine Savard, 2325 rue des Bibliothèques, Québec (QC), Canada G1V 0A6

Phone: 418 656-2131, ext. 12493

Fax : 418 656-3646

Email: karin.ensink@ psy.ulaval.ca 


\begin{abstract}
Background: There are significant gaps in our understanding of vulnerable and grandiose narcissism in adolescents and whether it is associated with psychosocial difficulties as well as risk factors such as child maltreatment. Objective: The aim of this study was to examine vulnerable and grandiose narcissism in adolescents and young adults. Method: 570 participants (ages 14-21) from the community completed an online survey. Vulnerable and grandiose narcissism were assessed with the Pathological Narcissism Inventory, while psycho-social difficulties were assessed with the Child Behaviour Checklist and maltreatment with the Childhood Experiences of Care and Abuse Questionnaire. Results: Gender-specific age trends were identified, with narcissism appearing to decrease with age for females, while increasing for males into early adulthood. For females, vulnerable and grandiose narcissism was associated with maltreatment and partially mediated the relationship between abuse and neglect and internalizing and externalizing difficulties. Conclusion: These findings underscore the importance of considering the relationship between childhood maltreatment experiences and suggest that the relationship between such experiences and adolescent psychosocial difficulties may be partially accounted for by the negative impact that maltreatment has on narcissism.
\end{abstract}

Keywords: Narcissism, abuse, gender, internalizing, externalizing. 


\section{INTRODUCTION}

There is a paucity of empirical data on pathological narcissism during adolescence and a lag in research on personality disorders in youth relative to adults (Barry \& Wallace, 2010). Maltreatment is a known risk factor for the development of pathological narcissism (Cater, Zeigler-Hill, \& Vonk, 2011; Maxwell \& Huprich, 2014), as well as other personality disorders (Besser, Zeigler-Hill, Pincus, \& Neria, 2013; Bifulco, Bernazzani, Moran, \& Jacobs, 2005; Chiesa \& Fonagy, 2014; Johnson, Cohen, Brown, Smailes, \& Bernstein, 1999), but this is understudied in adolescents (Mechanic \& Barry, 2014). Research has largely focused on adaptive and grandiose narcissism in adolescents considering that narcissism may include personality traits that are advantageous under certain circumstances (Jankowiak-Siuda, \& Zajkowski, 2013). Adaptive narcissism is considered central for successfully engaging with the developmental challenges of adolescence (Barry \& Wallace, 2010), while grandiose narcissism is associated with psychological difficulties and personality disorders (Barry \& Kauten, 2014; Fossati, Borroni, Eisenberg, \& Maffei, 2010; Marcinko et al., 2013). In contrast, vulnerable narcissism remains understudied. Vulnerable narcissism can be expected to be associated with difficulties in self-regulation and functioning, but this needs to be examined empirically. Little is also known regarding gender and age trends in vulnerable and grandiose narcissism during adolescence. The aim of this study is to summarize current theory within a psychodynamic framework and to review empirical studies on pathological narcissism as well as to provide preliminary data on vulnerable and grandiose narcissism, their relationships with age and gender during adolescence, and vulnerability factors such as childhood maltreatment. 


\section{What is Grandiose and Vulnerable Narcissism?}

Grandiose narcissism is characterised by arrogance and self-focused agentful and self-aggrandizing behaviour (Foster \& Brennan, 2012), dominance (Brown \& ZeiglerHill, 2004), hubris (Tracy, Chang, Robins \& Trzesniewsky, 2009), devaluation of others, deficient sensitivity to the concerns and social constraints of others, as well as a willingness to exploit them for personal gain (Morf \& Rhodewalt, 2001). On the other hand, vulnerable narcissism is characterised by a lack of self-confidence, contingent selfworth, reactivity to threats from others and a lack of dominance (Besser \& Priel, 2010). Both types of narcissism share an underlying concern with fulfilling their own needs rather than those of others.

In children and adolescents, grandiose narcissism has been linked to emotional and cognitive reactivity (Thomaes, Stegge, Olthof, Bushman, \& Nezlek, 2011), physical, verbal and relational aggression, as well as conduct problems (e.g. Golmaryami \& Barry 2009; Ha et al. 2008; Lau, Marsee, Kunimatsu, \& Fassnacht, 2011; Thomaes, Bushman, Orobio de Castro, Cohen, et al. 2009), proactive and reactive aggression (Fossati, Borroni, Eisenberg, \& Maffei, 2010), cyberbullying (Ang, Tan, \& Talib Mansor, 2011) and low peer preference (Barry et al. 2008). The maladaptive aspects of grandiose narcissism may not be evident when praise and affirmation is forthcoming, but manifests as rage when criticized (Bushman \& Baumeister, 1998) and socially excluded (Twenge \& Campbell, 2003), or as negative reactions when others have positive experiences (BaskinSommers, 2014). These difficulties are associated with deficits in self-observation (Baskin-Sommers, Crusemark \& Ronnigston, 2015) and emotional empathy (Fan, Wonneberger, Enzi, et al, 2010; Marissen, Dean \& Franken, 2012), and at a 
neurobiological level, with reduced gray matter in a region of the insula, involved in generating cortical representations of the experiences of others (Schulze, et al,, 2012).

\section{Kernberg's Conceptualization and Measurement of Narcissism}

Kernberg $(1985,2008)$ considers the presence of a grandiose self as the key feature of a narcissistic personality disorder. The grandiose self is seen to have developed as a protection from primitive affects of envy that are provoked when they perceive others as possessing all which they desire for themselves. This perception of others as 'having it all" provokes unbearable feelings of emptiness and worthlessness in the self. In contrast to the fluctuating nature of borderline personality disorder, narcissistic personalities are stable because they are organized around agency and achievement of self-goals, with interpersonal relationships used as a means of maintaining self-esteem (Campbell \& Green, 2007). Consistent with object-relations theory, Kernberg (1985, 2008) adds that to diagnose narcissistic pathology, one has to consider the quality of an individual's object relations and the pattern of their intra-psychic defensive system. Essentially, narcissistic individuals experience their relationships with others as exploitative and parasitic. They divide the world between those who have something valuable that they can obtain or extort and those who do not deserve consideration. They distinguish between extraordinary people on the one hand, with whom the association procure a sense of greatness themselves, and mediocre or worthless people on the other hand, with whom narcissistic individuals become obnoxious and abusive. In general, narcissistic individuals idealize the former and are contemptuous and devaluating of the latter. However, they also fear those they idealize, as they project onto them their own exploitative wishes, thus experiencing them as potentially dishonest and coercive. 
Therefore, they are unable to rely on any person and fear dependence on others, rendering all their object relations empty and dissatisfying.

More recently, Pincus and Lukowitsky (2010) demonstrated that distinct grandiose and vulnerable phenotypic profiles of pathological narcissism can be identified (Wright, Lukowitsky, Pincus, \& Conroy, 2010) using the Pathological Narcissism Inventory (PNI; Pincus et al. 2009), but to date this has largely been used with adults. A key distinction between the two phenotypes is that in grandiose narcissism aggression is directed towards others (Schoenleber et al., 2011), whereas in vulnerable narcissism aggression is directed towards self (Barry \& Kauten, 2014; Fossati, Borroni, Eisenberg, \& Maffei, 2010; Marcinko et al., 2013). However, this distinction is not always clear-cut. For example, in addition to proactive aggression, grandiose narcissism has also been shown to be associated with depression, anxiety and social stress in adolescents (Barry \& Kauten, 2014), and vulnerable narcissism with reactive aggression, in addition to depression (Fossati, Borroni, Eisenberg \& Maffei, 2010). Further investigation of the relationship between narcissistic phenotypes and internalizing and externalizing symptoms is thus warranted.

\section{Fonagy and Target's model of narcissism and failures in mentalizing}

From a psychodynamic mentalizing perspective, Fonagy and Target (2006)

propose that personality disorders are linked to early failures to engage with infants mentally, that is to make the mental effort to imagine their psychological experience and interpret and respond to their behaviour in terms of what these communicate about their underlying subjective experience, their mental states. They consider failures in early dyadic interpersonal processes to be the cause of narcissistic difficulties via their impact 
on the early development of a psychological sense of self and the capacity to accurately envision mental states in others, considered together as mentalizing. In the course of normal development, the early sense of self is seen as crystalized through dyadic interactions where the internal experience of the infant is reflected in the attitudes and actions of the parent. While this 'serve and return' dialogue between parent and infant happens at behavioural, embodied and emotional levels, Fonagy and Target (2006) consider interpersonal mentalizing, where the parent "sees", "minds" and "treats" the child in accordance with what they imagine and understand about their experience, as central for the "discovery" of the self in the mind of the other and the emergence of a robust capacity for mentalizing. They propose that in the course of normal development, parents and infants engage in a special type of communication, which operates at both verbal and non-verbal levels. During this communication, the infant's emotions, attitudes and intentions are mirrored, in a way that is marked, so that it can be recognized as similar to what is internally experienced, but also not quite the expression of the dispositional state of the parent - the mother expresses sadness but the crying infant is able to discern that it is not that she is feeling sad but that she is responding to his feeling of unhappiness. This process of mirroring, which of course can be difficult to achieve with some temperamentally hard-to-reach infants, facilitates the emergence of the early self and affect representations. The internalization of the parents' mirrored affect creates a second-order (or symbolic) representation of a constitutional state which not only helps regulate affect and other internal state through an understanding of internal experience, but also helps the child to appreciate the emotional experience of others through this improved awareness of his or her own subjective internal experience. In this way, the 
process of marked mirroring has an important function in helping the child move from an ego centric assumption that everyone shares their psychic reality, something Fonagy and Target (2006) refer to as psychic equivalence, to an emerging awareness that others have a similar, but also potentially slightly different, perspective. At early stages of development, all children universally assume psychic equivalence - their experience is shared by all: what they feel is how it is. When mature mentalizing is not fully achieved and the assumption of psychic equivalence is retained in too many interpersonal contexts, we encounter attitudes that are seen as characteristic of grandiose narcissists. According to Fonagy and Target (2006), the narcissist's unquestioning over-certainty about their perspectives as the only perspective, comes from the persistence of the mode of psychic equivalence in the mentalizing of such individuals so that they don't intuitively understand the relative nature of psychic reality and the fact that the experiences of others are never completely equivalent.

Narcissistic vulnerability is considered as rooted in failures in parental mirroring, where the parent is not mentally available or is unable to help the child discover themselves in the caregivers' minds because of the challenges the child presents. We all need to discover our thinking selves through the experience of feeling known by another. When the parent's reaction is unconnected to the child's expression, it is nevertheless internalized, and primes the child to develop an "alien self", a representation of self that is disconnected to their real self states. At the extreme, particularly in contexts where parents have themselves experienced abuse and neglect, the infant's distress triggers withdrawal and he or she is left with an experience of intolerable distress that cannot be known or shared with another. Emptiness and a dissociated self that has no traction with 
felt experience and which cannot support meaningful reactions and agentful action and is disconnected from the early experiential self leaves a vacuum where the representation of an agentive self should be. This may leave the child vulnerable to internalizing identities disconnected from self-experience including grandiose representations of the parent or they may defensively assume such representations in response to a sense of helplessness. These early vulnerabilities in self and mentalization may become more noticeable when early neglect is compounded by later trauma, particularly (but not invariably) in the context of attachment relationships, where the child may find himself or herself internalizing the destructive object in order to gain control over the experience of pain. He or she is also likely to lose trust regarding the benign intentions of the attachment figure and fears instead to engage with their minds, so that they defensively shut off the only likely sources of assistance: a reflective mind which could (through contingent marked mirroring) help them make sense of their confusing inner experience (Fonagy, Gergely, Jurist \& Target, 2002).

In sum, grandiose narcissism is hypothesized to be associated with a vulnerability to using prementalizing modes where the distinct individual nature of psychic reality is not grasped intuitively. This limits interactions with others and the ability to consider and engage with their experience and appreciate that others have different perspectives. Vulnerable narcissism, on the other hand, is associated with an inadequately developed fragile self that lacks coherence and a false or alien self that is insufficiently connected to physical (actual) experience. This model contributes a way of thinking about narcissism and associated self and interpersonal difficulties in terms of failures in thinking and 
mentalizing that impact on the capacity to interpret interpersonal reactions adequately and leaves the self as unknown and disconnected to others.

\section{Narcissism and Childhood Histories of Abuse and Neglect: Research and Theory}

Maltreatment is recognized as a key factor increasing the risk for all types of psychiatric disorders (Cicchetti \& Banny, 2014; Dorahy, Middleton, Seager, Williams, \& Chambers, 2016; Dvir, Ford, Hill, \& Frazier, 2014). This appears to be the case for depressive and anxiety disorders, as well as for personality disorders such as BPD and non-suicidal self injury (Bégin et al., accepted), and psychotic disorders such as schizophrenia, which until recently, was considered as primarily genetically determined (Berthelot, Paccalet, et al., 2015). Early trauma, such as child abuse and neglect, as well as harsh or affectionless parenting, is considered an important stress factor that undermines and disrupts normal development of self- and affect regulation. Furthermore, it acts as a general diathesis which can activate underlying genetic risks for psychopathology. It is also associated with decreased heart rate variability, higher levels of stress hormones, and reduced or impaired immune response (Van der Kolk, 2016). There is some evidence of an association between narcissism in adults and childhood maltreatment (Cater, Zeigler-Hill, \& Vonk, 2011; Maxwell \& Huprich, 2014), but also with the other extreme of excessive parental admiration (Otway \& Vignoles, 2006). Similar research in adolescence is absent, although some associations have been found with poor monitoring and discipline (Mechanic \& Barry, 2014), consistent with a diathesis-stress model where non-optimal parenting undermines healthy self-development in interaction with temperament in the development of narcissism (Thomaes et al., 2013). In the absence of parenting that can support the healthy development of self, narcissistic 
vulnerability is a predictable outcome, or, an unrealistic and inflated sense of self may evolve at the same time to bolster the self and inspire the belief in one's capacity and agency to succeed and survive.

From an attachment perspective, Meyer and Pilkonis (2011) propose that grandiose narcissism is prototypically associated with dismissive attachment where there is an inflated representation of the self and an experience of being superior yet unacknowledged, while others are perceived as inferior and withholding deserved admiration. The basic premise is that identification with caring attachment figures promotes the child's belief that they themselves embody the strength and goodness initially provided by attachment figures (Mikulincer \& Shaver, 2016). This fosters a healthy self-love and narcissism that is neither overly fragile nor grandiose, and facilitates a sense of coherence, a realistic sense of self-standards and protects from overly harsh self-criticism, thereby preserving clear mindedness even in the face of threatening circumstances. In contrast, lack of parental responsiveness and parental rejection is considered to contribute to vulnerable and unstable self-esteem and disorders

of the self, because the child never develops the sense that love and esteem of significant others can be assumed. Other pathways, such as that involving overindulgence, are also considered to contribute to grandiose narcissism. Overindulgence is thought to install a mental model in which the self is considered as indiscriminately worthy of attention and praise, regardless of behaviour, while representations of others are impoverished and essentially seen in terms of serving the needs of the self, rather than as autonomous agents.

\section{Pathological Narcissism, Age and Gender}


With regard to age, preliminary evidence suggests that grandiose narcissism increases significantly from 14 to 18 years of age and decreases (non-significantly) from age 18 to 23, (Carlson \& Gjerde, 2009) before further declining and eventually plateauing during adulthood (Foster et al., 2003; Wilson \& Sibley, 2011). This suggests that narcissism may be solicited at specific times during the life cycle and specifically in the context of developmental challenges at the entry into adulthood when adolescents face multiple challenges in the course of finding their way independently in the adult world, succeeding academically, finding work and becoming financially and socially independent.

Findings from a meta-analytic review of gender differences in narcissism conclude that men tend to score higher on the exploitation and entitlement facet as well as the leadership and authority facet of grandiose narcissism, however no gender-related differences for vulnerable narcissism were found (Grijalva, et al., 2015). Other studies suggest that while men scored higher on exploitation and grandiose fantasy subscales of the PNI (Wright and colleagues, 2010), women scored higher on scales indicative of vulnerable narcissism, such as contingent self-esteem, devaluing, and hiding the self. Yet little is known regarding gender-specific age trends for vulnerable and grandiose narcissism in adolescence.

The aim of this study is to investigate grandiose and vulnerable narcissism during adolescence including age and gender effects, and to explore its association with internalizing and externalizing difficulties, as well as with potential risk factors such as childhood experiences of abuse and neglect. We consider that it is pertinent to investigate these relationships in different populations such as adolescents in the community, 
adolescents in clinical settings, as well as adolescents who have experienced severe abuse and neglect. In the first instance, we were interested in examining how this presents in adolescents in the community. Therefore, the first objective of this study was to examine age and gender effects in the development of pathological narcissism during adolescence. Based on previous findings (Grijalva, et al., 2015), we expected that adolescent boys would have higher grandiose narcissism scores and that adolescent girls would have higher scores for vulnerable narcissism. Furthermore, we expected an increase in grandiose narcissism between the ages of 14 to 18 . We had no specific hypothesis regarding age trends and vulnerable narcissism, given the absence of previous findings in this regard. The second objective was to examine the relationship between pathological narcissism and internalizing and externalizing difficulties. Based on the literature (Barry \& Kauten, 2014, Fossati, Borroni, Eisenberg \& Maffei, 2010), we expected that higher levels of vulnerable and grandiose narcissism would be associated with more internalizing and externalizing difficulties, with a stronger relationship between grandiose narcissism and externalizing difficulties, and vulnerable narcissism and internalizing difficulties. The third objective was to investigate the association between abuse and neglect and pathological narcissism. Considering the findings from previous studies (Mechanic \& Barry, 2014; Otway \& Vignoles, 2006), both types of pathological narcissism were expected to be associated with parental coldness, including antipathy, neglect and psychological abuse. A final objective was to examine the hypothesis that the relationship between abuse and internalizing and externalizing symptoms may be partially mediated by pathological narcissism, depending on the associations found. 


\section{METHOD}

\section{Sample and Participant Selection:}

570 adolescents aged 14 to 21 years old (426 females, 138 males, 6 not specified, $\mathrm{M}=17.91, \mathrm{SD}=2.99)$ were recruited from high schools $(48.2 \%)$ and a university $(51.8 \%)$ in a French-Canadian city and surrounding area so that the sample represents a wide socio-economic range. Participants were principally of Caucasian descent $(91.3 \%)$, while the remainder were Asian (2.5\%), African American (1.5\%) or reported other ethnicities (4.7\%). Participants were invited to complete online questionnaires via a secured platform. This platform included a description of the study, a consent page, and all questionnaires in a user-friendly online format. Each questionnaire was displayed on a unique webpage and proceeding to a subsequent questionnaire was permitted only once all questions in the current questionnaire had been answered. All items were in multiplechoice format. Ethical approval was obtained from the University Laval ethics committee, project number 2013-201.

\section{Assessment and Measures}

Pathological Narcissism Inventory for Adolescents (PNI-A). This study used the French version of the Pathological Narcissism Inventory (PNI; Pincus et al., 2009) adapted for adolescents (Chrétien, Ensink, Descoteaux, Daigle, Normandin, submitted). The PNI-A is comprised of 52 items scored using a 6-point Likert scale. These items cover the following seven scales: exploitative (EXP, $\alpha=.81$ ), self-sacrificing selfenhancement (SSSE, $\alpha=.79$ ), grandiose fantasy (GF, $\alpha=.71)$, entitlement rage (ER, $\alpha=.82$ ), contingent self-esteem (CSE, $\alpha=.90)$, hiding the self (HS, $\alpha=.66$ ) and devaluation (DEV, $\alpha=.82$ ) scales (author reference). The French PNI-A has been shown to have a 
robust factor structure, good test-retest reliability, and good construct validity. In addition, the same two-factor structure representing grandiose and vulnerable narcissism demonstrated to be present for the adult PNI was replicated in the French PNI-A.

Child Behavior Checklist - Youth Self Report (CBCL-YSR). In the current study the French version of the Child Behavior Checklist - Youth Self Report 11-18 (Achenbach, 1991) was used to measure internalizing and externalizing difficulties in adolescents. The YSR contains 112 items and responses are rated on a three-point scale, 1) "does not apply", 2) "sometimes" and 3) "often or always". The internalizing scale of the YSR consists of $(\alpha=.90$, anxiety/depression; $\alpha=.84$, withdrawal/depression; $\alpha=.71$, and somatic complaints subscales; $\alpha=.80$ ), while the externalizing scale comprises the ( $\alpha=.90$, rule-breaking; $\alpha=.81$ and aggressive behaviour subscales; $\alpha=.86$ ). The CBCL YSR is widely used to assess internalizing and externalizing in adolescents and has been validated in a variety of languages (Achenbach \& Rescorla, 2001). The same factor structure was replicated with the French version and confirmed that the YSR internalizing and externalizing subscales measured distinct dimensions of difficulties in youth (Song, Singh \& Singer, 1994). The internal consistency of the internalizing and externalizing subscales in the present study were .92 and .83 , respectively.

Childhood Experiences of Care and Abuse Questionnaire (CECA-Q). The French version of the CECA-Q was used in the present study. The CECA-Q (Smith, Lam, Bifulco, \& Checkley, 2002) is a 128 item self-report questionnaire used to assess lack of parental care (antipathy and neglect), as well as physical, psychological, and sexual abuse, before the age of 17 by both the mother and father. The antipathy subscale assesses hostile, cold, rejecting or blaming attitudes from parents (i.e. makes the child a 
scapegoat for family issues). The neglect subscale includes questions that assess the lack of provision of physical, educational, and emotional needs. The physical abuse subscale refers to hitting of different intensities and frequencies. The psychological abuse subscale assesses threatening or cruel behaviours as well as isolation. Finally, the sexual abuse subscale assesses experiences of sexual contact with an adult, or propositions by them or exposure to situations that are of a sexual nature but excludes consensual acts between peers. A composite score of abuse can also be computed. The CECA-Q showed high internal consistency for care scales, high test re-test reliability for all scales, and good convergent validity with an established measure of parental care (Bifulco, Bernazzani, Moran, \& Jacobs, 2005). The internal consistency coefficients for this study were .85, $.84, .79, .88$ and .91 for all the subscales including antipathy, neglect, physical abuse, psychological abuse, and sexual abuse subscales, respectively.

Self-Perception Profile for Adolescents (SPPA). The SPPA is a 45-item measure of adolescents' self-concept in terms of personal satisfaction and feelings of selfefficacy developed by (Harter, 1988). In this study the French version (Bouffard, et al., 2002) was used. Participants are asked to indicate which type of person they are most like and then whether the description is "sort of true" or "really true" of him or her. Each question is scored from 1 to 4 , (where a score of 1 signifies relatively low perceived competence, while a score of 4 suggests a high-perceived competence). The SPPA is comprised of subscales that assess self-perception in different areas including school $(\alpha=.77)$, social acceptance $(\alpha=.83)$, athleticism $(\alpha=.91)$, physical appearance $(\alpha=.91)$, job competence $(\alpha=.73)$, romantic appeal $(\alpha=.63)$, behavioural conduct $(\alpha=.76)$, close friendships $(\alpha=.84)$ and self-esteem $(\alpha=.85)$. The factor structure and internal consistency 
of the French version were shown to correspond to those of the original English version in a community sample (Bouffard et al., 2002). In addition, the test-retest reliability of the translated version was also shown to be good (Bouffard et al., 2002). In the present study, only the aggregate score of overall self-esteem was used $(\alpha=.91)$.

Data Analytic Strategy. Gender related differences in vulnerable and grandiose narcissism were examined using $t$-tests. We also examined whether gender moderated the relationship between age and vulnerable and grandiose narcissism. The presence of an interaction between age and gender on pathological narcissism was tested using linear regression models. Then, correlations were used to examine relationships between vulnerable and grandiose narcissism, internalizing and externalizing difficulties, as well as aversive childhood experiences. To examine the contribution of different types of aversive childhood experiences in the prediction of vulnerable and grandiose narcissism linear regression analyses were conducted. Finally, mediation analyses were performed to examine whether vulnerable and grandiose narcissism explained the relationship between abuse and internalizing and externalizing symptoms. All analyses were conducted using IBM Statistics Package for the Social Science (SPSS) v.23 and MPlus 1.4.

\section{RESULTS}

Preliminary analyses. Q-Q plots were visually inspected to assure that assumptions of normality were respected.

Age and gender effects. T-tests were performed to compare means for grandiose and vulnerable narcissism by gender. Boys had significantly higher scores for grandiose 
narcissism, $t(192)=3.76, p<.001$, and girls had significantly higher scores for vulnerable narcissism, $t(203)=2.59, p<.05$.

Linear regression models were used to test for an interaction between age and gender on pathological narcissism. A significant interaction between age and gender was found for both grandiose and vulnerable subtypes, as well as for pathological narcissism overall $(b=.127, p<.01, b=.146, p<.01$ and $\mathrm{b}=.138, \mathrm{p}<.01$, respectively $)$, indicating that pathological narcissism is differentially expressed between genders depending on age.

Next, correlations were used to examine the direction and strength of the association between age and pathological narcissism, stratified by gender. The results are presented in Table 1. For girls, age was negatively correlated with grandiose and vulnerable narcissism and PNI total score. For boys, age was positively correlated with the two phenotypes and with PNI total score. To verify that this age trend was specific to narcissism and not a general age trend related to self-esteem, we examined the relationship between self-esteem and age, which was subsequently found to be nonsignificant for females and males.

Pathological narcissism, internalizing and externalizing difficulties. For girls, there were significant correlations between vulnerable narcissism and internalizing $(r=$ $.631, p<.001)$ and externalizing difficulties $(r=.544, p<.001)$, as well as between grandiose narcissism and internalizing $(r=.315, p<.001)$ and externalizing difficulties $(r$ $=.408, p<.001)$. For boys, there were significant correlations between vulnerable 
narcissism and internalizing $(r=.492, p<.01)$ and externalizing difficulties $(r=.437, p$ $<.01)$ only.

Narcissism and maltreatment. Among girls, vulnerable and grandiose narcissism were significantly correlated with antipathy, $(r=.489, p<.001$ and $r=.311, p$ $<.001$, respectively), neglect $(r=.452, p<.001$ and $r=.242, p<.001$, respectively), psychological abuse $(r=.346, p<.001$ and $r=.213, p<.01$, respectively), physical abuse $(r=.148, p=.061$ and $r=.215, p<.01$, respectively) and sexual abuse $(r=.164, p$ $<.05$ and $r=.169, p<.05$, respectively). In boys, there were no significant correlations between abuse and narcissism.

\section{Narcissism, maltreatment, and internalizing and externalizing difficulties.}

Mediation analyses were conducted to investigate the potential mediating role of pathological narcissism in the development of internalizing and externalizing difficulties in the context of maltreatment. Internalizing and externalizing difficulties were the dependent variables, a composite score of all types of maltreatment was the independent variable, and grandiose and vulnerable narcissism were the mediating variables. Results from the mediation analyses are shown in Figures 1, 2 and 3. For girls, vulnerable narcissism had a significant indirect effect on the relationship between maltreatment and internalizing difficulties, $a b=.232,95 \%$ CI [2.515, 5.959], $\mathrm{P}_{\mathrm{M}}=.762$, and externalizing difficulties, $a b=.202,95 \%$ CI [1.523, 4.047], $\mathrm{P}_{\mathrm{M}}=.872$. Also for girls, grandiose narcissism had a significant indirect effect on the association between maltreatment and externalizing difficulties only, $a b=.096,95 \%$ CI [0.525, 2.322], $\mathrm{P}_{\mathrm{M}}=.285$. 


\section{DISCUSSION}

The aim of this study was to determine if it is possible to reliably measure pathological narcissism during adolescence and to explore the relationships with age, gender and childhood maltreatment. A secondary objective was to examine the possible role of narcissism as a mediator of the relationship between abuse and internalizing and externalizing difficulties.

Firstly, the findings show that it is possible to measure grandiose and vulnerable narcissism in adolescents and that narcissism is sensitive to age and gender. Gender moderated the relationship between age and pathological narcissism, with genderstratified analyses revealing two distinct age trends for pathological narcissism. Both vulnerable and grandiose narcissism decrease with age in adolescent girls, while increasing in adolescent boys between the ages of 14 to 21 . Considering the crosssectional nature of the data, further longitudinal research is necessary to confirm these age trends. Nonetheless, the findings suggest that there may be distinct age-related pressures for adolescent boys and girls and gender-specific responses and experiences of personal and interpersonal challenges. For example, given the importance of physical appearance for adolescent self-esteem, puberty has been said to distance girls from their ideal bodies, while uniting boys with theirs (Seidah, Bouffard \& Vezeau, 2004). Along these lines, when younger adolescent girls are challenged to adapt to and integrate their changing bodies during puberty, they may be particularly vulnerable or resort to grandiose strategies, yet may be able to develop healthier narcissistic integration with time. On the other hand, qualities associated with grandiose narcissism, such as being autonomous and dominant, are associated with success and social prestige in young men, 
(Freedman, 1984; Grijalva, Newman, Tay, Donnellan, Harms, Robins, \& Yan, 2015;

Zhou et al., 2012) and such rewards may underlie the trend observed where grandiose narcissism appears to increase with age into young adulthood. However, there was also an increase in vulnerable narcissism in adolescent boys. This may suggest that the challenges of emerging adulthood, like leaving home and engaging with new work and/or academic responsibilities and social demands for example, may contribute to a sense of vulnerability and self doubt that is defensively responded to with either inflated narcissism or increasing self doubt and vulnerable narcissism.

Vulnerable narcissism was associated with both internalizing and externalizing difficulties, with strong correlations for adolescent girls and medium strength correlations for adolescent boys. For both genders, specific dimensions of vulnerable narcissism, namely hiding the self, contingent self-esteem, devaluation and entitlement rage, appeared to be most associated with internalizing and externalizing difficulties. This suggests that these maladaptive self-processes are associated with an increased risk of difficulties in self regulation and adaptation. Grandiose narcissism was weakly correlated with internalizing difficulties and moderately correlated with externalizing difficulties in adolescent girls, extending previous findings linking grandiose narcissism with internalizing symptoms in girls (Malkin, Barry \& Zeigler-Hill, 2011). Interestingly, for adolescent boys, grandiose narcissism did not appear to be associated with either internalizing or externalizing difficulties. This is broadly in line with previous findings that some grandiose traits may have adaptive advantages (Jankowiak-Siuda, Zajkowski, 2013; Obradovic, 2010), but this appears to be the case for boys only. These findings of gender-specific relationships nuance previous findings linking vulnerable and grandiose 
narcissism with internalizing and externalizing symptoms during adolescence in general (Barry \& Kauten, 2014; Barry et al., 2015). It appears that finding the right balance in terms of narcissism may be particularly challenging and important for girls and that there are considerable costs associated with both vulnerable and grandiose styles.

Furthermore, the findings show that narcissism was associated with maltreatment, but only in girls. For girls, neglectful parenting involving antipathy, psychological abuse and neglect, and also the experiences of physical or sexual abuse, was significantly positively correlated with vulnerable and grandiose narcissism. These findings extend and nuance previous findings linking pathological narcissism in adults with parental indifference (Otway \& Vignoles, 2006) and abuse and neglect (Maxwell \& Huprich, 2014), as well as findings linking personality disorders like borderline personality disorder with physical and sexual abuse (Fossati, Madeddu, \& Maffei, 1999; Waxman, Fenton, Skodol, Grant, \& Hasin 2013; Zanarini et al., 1997). Curiously, these relations were only observed in girls, suggesting that girls are more vulnerable to the effect of inadequate parenting on the developing self. This is in line with similar findings suggesting that girls are more vulnerable to the impact of abuse and antipathy (Schilling, Aseltine, \& Gore, 2007; Scimmenti \& Bifulco, 2015; Widom, Czaja, \& Dutton, 2008). For girls, both grandiose and vulnerable narcissism mediated the relationship between an aggregate score of neglect and abuse, and internalizing and externalizing difficulties, suggesting that girls may be more vulnerable than boys in terms of neglect and abuse affecting core self processes and undermining the development of healthy narcissism that can facilitate self-regulation. In terms of the diathesis-stress model proposed by Thomaes and collaborators (2013), it appears that for females, being exposed to maltreatment may 
be an important environmental factor that undermines the development of normal narcissism, and increases the underlying risk for vulnerable or grandiose narcissism.

When we consider the clinical implications of these findings, it points to the importance of integrating an awareness and focus on narcissism in work with adolescents, especially adolescent girls who have experienced antipathy and other forms of maltreatment. Adolescence is likely to be a particularly important time for benefitting from therapeutic help to develop an awareness of grandiose strategies, as well address vulnerabilities, and facilitate the emergence of healthy self awareness and a realistic appreciation of their own strengths and limitations, as well as that of others. Maltreatment-related difficulties with basic trust and the sense that the fact that adolescents with maltreatment experiences may feel different from peers without such experiences, may also further contribute to grandiose styles where others are devaluated and considered as untrustworthy and unable to provide the desired for understanding. Narcissism and self processes associated with healthy and pathological narcissism is likely a key dimension underlying psychopathology at adolescence, with long term implications for adaptive and maladaptive functioning, and a better understanding and more effective interventions designed to address difficulties at this level, should be a priority.

From a theoretical perspective, the strength of the relationship between neglectful, psychologically absent and abusive parenting and narcissism is in line with Fonagy and Target's (2006) developmental model. Narcissistic difficulties are understood as linked to early failures in dyadic parenting interactions, where the young child is deprived of caregiving relationships in which they have the opportunity to discover their minds in the 
mind of the parent. Fonagy and Target (2006) consider the principal tragedy characterizing narcissism, as the difficulty in considering the psychic experiences of others, because they predominantly use prementalizing modes where their own psychic reality and thoughts are considered as reality and they are unable to take into consideration the perspectives and differing psychic experience of others. Whereas Fonagy and Target elucidate the failures in mentalizing and interpersonal interpretative processes, Kernberg's $(1985,2008)$ model complements this emphasis on mentalization by describing the distorted perceptions of others that account for the characteristic interpersonal difficulties associated with narcissism. For a child who was not invested in and treasured by the caregiver, or worse, experienced maltreatment, the fear, aggression, and general negative affect activated by such experiences contribute to a predominance of negative affect which distorts the way relationships are perceived. In Kernberg's (1985; 2008) model, intense negative affect that originates from early dyadic failures, where the child is left without being seen for who they are, activates primitive envy that renders mentalization impossible so that it is impossible to appreciate others' strengths, difficulties and concerns.

While this study has several strengths including its relatively large sample size of adolescents and young adults from the community and the assessment of both grandiose and vulnerable dimensions of narcissism, some limitations need to be considered before generalising the findings. The cross-sectional nature of the study is a limitation and age effects ideally need to be examined using a longitudinal design. Furthermore, the use of self-report measures and retrospective reports of maltreatment can be seen to be a limitation, although there is convergent evidence that self-reports of maltreatment 
experiences can be considered as reliable. The findings regarding the relationships between maltreatment and narcissism also need to be replicated in future studies and the mental and psychological mechanisms underlying these associations as suggested by the models of Fonagy and Target (2006) and Kernberg (1985; 2008), need further investigation.

Narcissism during adolescence appears to follow gender-specific patterns where both grandiose and vulnerable types increase with age into early adulthood for males while decreasing with age for females. Inadequate development of the self, manifested as vulnerable narcissism, is associated with psychological difficulties of both an internalizing and externalizing type, irrespective of gender. Grandiose narcissism was also associated with such difficulties, but only for girls. Furthermore, the findings revealed links between maltreatment and vulnerable and grandiose narcissism in girls and the relationships were partially mediated by narcissism. The findings point to the importance of considering early experiences of maltreatment in our understanding and treatment of adolescent narcissism and suggest that girls may face particular challenges in terms of the development of adaptive narcissism and pay a high price when vulnerable or grandiose patterns evolve. In sum, failures in developing a realistic healthy sense of self and narcissism that is neither fragile nor overly grandiose, may be at the root of psychological difficulties and addressing these frequently overlooked underlying difficulties, is likely particularly important for adolescents to face the challenges inherent in this period and redress maladaptive personality processes. 


\section{Conflict of interest}

K.E., S.C., D.D., L.N. and P.F have no conflicts of interest to disclose. 


\section{References}

Achenbach, T. M. (1991). Manual for the YSR and 1991 profile. Burlington, VT: University of Vermont, Department of Psychiatry.

Achenbach, T. M., \& Rescorla, L. A. (2001). Manual for the ASEBA School-Age Forms \& Profiles. Burlington, VT: University of Vermont, Research Center for Children, Youth, \& Families.

Ang, R. P., Tan, K. A., \& Mansor, A. T. (2010). Normative beliefs about aggression as a mediator of narcissistic exploitativeness and cyberbullying. Journal of Interpersonal Violence, 0886260510388286.

Barry, C.T., \& Kauten, R.L. (2014). Nonpathological and pathological narcissism: which self-reported characteristics are most problematic in adolescents? Journal of Personality Assessment, 96(2), 212-219.

Barry, C.T., \& Wallace, M.T. (2010). Current considerations in the assessment of youth narcissism: indicators of pathological and normative development. Journal of Psychopathology and Behavioral Assessment, 32(4), 479-489.

Barry, T. D., Barry, C. T., Deming, A. M., \& Lochman, J. E. (2008). Stability of psychopathic characteristics in childhood: the influence of social relationships. Criminal Justice and Behavior, 35, 244-262.

Baskin-Sommers, A., Krusemark, E., \& Ronningstam, E. (2014). Empathy in Narcissistic Personality Disorder: From Clinical and Empirical Perspectives. Personality Disorders, 5(3), 323-333. 
Bégin et al., submitted

Berthelot, N., Paccalet, T., Gilbert, E., Moreau, I., Mérette, C., Gingras, N., ... \& Maziade, M. (2015). Childhood abuse and neglect may induce deficits in cognitive precursors of psychosis in high-risk children. Journal of psychiatry \& neuroscience: $J P N, 40(5), 336$.

Besser, A., Zeigler-Hill, V., Pincus, A.L., \& Neria, Y. (2013). Pathological narcissism and acute anxiety symptoms after trauma: a study of Israeli civilians exposed to war. Psychiatry, 76(4), 381-397.

Besser, A., \& Priel, B. (2010). Grandiose narcissism versus vulnerable narcissism in threatening situations: Emotional reactions to achievement failure and interpersonal rejection. Journal of Social and Clinical Psychology, 29(8), 874.

Bifulco, A., Bernazzani, O., Moran, P. M., \& Jacobs, C. (2005). The Childhood Experience of Care and Abuse Questionnaire (CECA.Q): validation in a community series. British Journal of Clinical Psychology, 44, 563-581.

Bouffard, T., Seidah, A., McIntyre, M., Boivin, M., Vezeau, C., \& Cantin, S. (2002). Mesure de l'estime de soi à l'adolescence : version canadienne-française du SelfPerception Profile for Adolescents de Harter. Revue Canadienne des Sciences du Comportements, 34(3), 158-162.

Brown, R. P. \& Zeigler-Hill, V. (2004). Narcissism and the non-equivalence of selfesteem measures: A matter of dominance? Journal of Research in Personality, 38(6), 585-592. 
Campbell, W. K., \& Green, J. D. (2007). Narcissism and interpersonal self-regulation. In Wood, J. V., Tesser, A. \& Holmes, J. G. (Eds.), Self and Relationships (pp. 7394). New York, NY: Psychology Press.

Carlson, K.S., \& Gjerde, P.F. (2009). Preschool personality antecedents of narcissism in adolescence and young adulthood: A 20-year longitudinal study. Journal of Research in Personality, 43, 570-578.

Cater, T.E., Zeigler-Hill, V., \& Vonk, J. (2011). Narcissism and recollections of early life experiences. Personality and Individual Differences, 51, 935-939.

Cicchetti, D., \& Banny, A. (2014). A developmental psychopathology perspective on child maltreatment. In M. Lewis \& K. Rudolph (Eds.), Handbook of developmental psychopathology (pp. 723-741). New York: Springer.

Chiesa, M., \& Fonagy, P. (2014). Reflective function as a mediator between childhood adversity, personality disorder and symptom distress. Personality and Mental Health, 8, 52-66.

Chrétien, S., Ensink, K., Descoteaux, J., Daigle, D., \& Normandin, N. (submitted). Validation of the French version of the Pathological Narcissism Inventory for adolescents. Journal of Adolescent Research.

Dorahy, M. J., Middleton, W., Seager, L., Williams, M., \& Chambers, R. (2016). Child abuse and neglect in complex dissociative disorder, abuse-related chronic PTSD, and mixed psychiatric samples. Journal of Trauma \& Dissociation, 17(2), 223236. 
Dvir, Y., Ford, J. D., Hill, M., \& Frazier, J. A. (2014). Childhood maltreatment, emotional dysregulation, and psychiatric comorbidities. Harvard review of psychiatry, 22(3), 149.

Author Reference. (Submitted). Validation of the Narcissistic Personality Inventory for Adolescents. Personality Assessment.

Fan Y, Wonneberger C, Enzi B, de Greck M, Ulrich C, Tempelmann C, Bogerts B, Doering S, Northoff G. (2011). The narcissistic self and its psychological and neural correlates: an exploratory fMRI study. Psychological Medecine, 41(8), 1641-50.

Fonagy, P., Gergely, G., Jurist, E., \& Target, M. (2002). Affect regulation, mentalization, and the development of the self. New York: Other Press.

Fonagy, P., \& Target, M. (2006). The mentalization-focused approach to self pathology. Journal of personality disorders, 20(6), 544.

Fossati, A., Borroni, S., Eisenberg, N., \& Maffei, C. (2010). Relations of proactive and reactive dimensions of aggression to overt and covert narcissism in nonclinical adolescents. Aggressive Behavior, 36, 21-27.

Fossati, A., Madeddu, F., \& Maffei, C. (1999). Borderline personality disorder and childhood sexual abuse: A meta-analytic study. Journal of Personality Disorders, $13,268-280$.

Foster, J. D. \& Brennan, J. C. (2011) Narcissism, the Agency Model, and ApproachAvoidance Motivation, in The Handbook of Narcissism and Narcissistic 
Personality Disorder: Theoretical Approaches, Empirical Findings, and Treatments (eds W. K. Campbell and J. D. Miller), John Wiley \& Sons, Inc., Hoboken, New Jersey.

Foster, J. D., Campbell, W.K., \& Twenge, J.M. (2003). Individual differences in narcissism: Inflated self-views across the lifespan and around the world. Journal of Research in Personality, 37, 469-486.

Freedman, R. J. (1984). Reflections on beauty as it relates to health in adolescent females. Women and Health, 9(2-3), 29-45.

Golmaryami, F. N., \& Barry, C. T. (2009). The associations of self-reported and peerreported relational aggression with narcissism and self-esteem among adolescents in a residential setting. Journal of Clinical Child \& Adolescent Psychology, 39(1), 128-133.

Grijalva, E., Newman, D. A., Tay, L., Donnellan, M .B., Harms, P. D., Robins, R. W., \& Yan, T. (2015). Gender differences in narcissism: A meta-analytic review. Psychological Bulletin, 141(2), 261-310.

Ha, C., Petersen, N., \& Sharp, C. (2008). Narcissism, self-esteem, and conduct problems. European child \& adolescent psychiatry, 17(7), 406-413.

Harter, S. (1988). Manual for the Self-Perception Profile for Adolescents. University of Denver. 
Jankowiak-Siuda, K., \& Zajkowski, W. (2013). A neural model of mechanisms of empathy deficits in narcissism. Medical Science Monitor : International Medical Journal of Experimental and Clinical Research, 19, 934-941.

Johnson, J.G., Cohen, P., Brown, J., Smailes, E.M., \& Bernstein, D.P. (1999). Childhood maltreatment increases risk for personality disorders during early adulthood. Archives of General Psychiatry, 56, 600-606.

Kernberg, O. F. (1985). Borderline conditions and pathological narcissism. New York: Rowman \& Littlefield Publishers, Inc.

Kernberg, O. (2008). Aggressivity, Narcissism, and Self-Destructiveness in the Psychotherapeutic Relationship: New Developments in the Psychopathology and Psychotherapy of Severe Personality Disorders. Yale University Press.

Lau, K.S.L., Marsee, M.A., Kunimatsu, M.M., \& Fassnacht, G.M. (2011). Examining associations between narcissism, behavior problems, and anxiety in non-referred adolescents. Child Youth Care Forum, 40, 163-176.

Malkin, M.L., Barry, C.T., \& Zeigler-Hill, V. (2011). Covert narcissism as a predictor of internalizing symptoms after performance feedback in adolescents. Personality and Individual Differences, 51, 623-628.

Marcinko, D., Jaksic, N., Ivezic, E., Skocic, M., Suranyi, Z., Loncar, M., Franic, T., \& Jakovljeliv, M. (2013). Pathological narcissism and depressive symptoms in psychiatric outpatients: mediating role of dysfunctional attitudes. Journal of Clinical Psychology, 70(4), 341-352. 
Marissen, M. A., Deen, M. L., Franken, I. H. (2012). Disturbed emotion recognition in patients with narcissistic personality disorder. Psychiatry Research, 198(2), 26973.

Maxwell, K., \& Huprich, S. (2014). Retrospective reports of attachment disruptions, parental abuse and neglect mediate the relationship between pathological narcissism and self-esteem. Personality and Mental Health, 8, 290-305.

Mechanic, K.L., \& Barry, C.T. (2014). Adolescent grandiose and vulnerable narcissism: associations with perceived parenting practices. Journal of Child and Family Studies, 24, 1510-1518.

Meyer, B., \& Pilkonis, P. A. (2011). Attachment Theory and Narcissistic Personality Disorder, in The Handbook of Narcissism and Narcissistic Personality Disorder: Theoretical Approaches, Empirical Findings, and Treatments (eds W. K. Campbell and J. D. Miller), John Wiley \& Sons, Inc., Hoboken, New Jersey.

Mikulincer, M. \& Shaver, P. R. (2016). Attachment in adulthood: structure dynamics and change ( $2^{\text {nd }}$ Ed.). New York: The Guildford Press.

Morf, C.zsù C., \& Rhodewalt, F. (2001). Unraveling the paradoxes of narcissism: A dynamic self-regulatory processing model. Psychological Inquiry, 12(4), 177-196.

Obradovic, J., Burt, K. B., \& Masten, A. S. (2010). Testing a dual cascade model linking competence and symptoms over 20 years from childhood to adulthood. Journal of Clinical Child and Adolescent Psychology, 39, 90-102. 
Otway, L.J., \& Vignoles, V.L. (2006). Narcissism and childhood recollections: a quantitative test of psychoanalytic predictions. Personality and Social Psychology Bulletin, 32(1), 104-116.

Pincus, A. L., Ansell. E. B., Pimentel, C. A., Cain, N. M., Wright, A. G. C., Levy, K. N. (2009). Initial construction and validation of the Pathological Narcissism Inventory. Psychol. Assess. 21, 365-379.

Pincus, A. L., \& Lukowitsky, M. R. (2010). Pathological narcissism and narcissistic personality disorder. Annual Review of Clinical Psychology, 6, 421-446.

Schilling, E. A., Aseltine, R. H., \& Gore, S. (2007). Adverse childhood experiences and mental health in young adults: a longitudinal survey. BMC public health, 7(1), 30.

Schimmenti, A., \& Bifulco, A. (2015). Linking lack of care in childhood to anxiety disorders in emerging adulthood: the role of attachment styles. Child and Adolescent Mental Health, 20(1), 41-48.

Schoenleber, M., Sadeh, N., \& Verona, E. (2011). Parallel syndromes: Two dimensions of narcissism and the facets of psychopathic personality in criminally involved individuals. Personality Disorders: Theory, Research, and Treatment, 2(2), 113127.

Schulze, L., Dziobek, I., Vater, A., Heekeren, H. R., Bajbouj, M., Renneberg, B., Heuser, I., Roepke, S. (2013). Gray matter abnormalities in patients with narcissistic personality disorder. Journal of Psychiatric Research, 47(10), 1363-9. 
Seidah, A., Bouffard, T., \& Vezeau, C. (2004). Perceptions de soi à l'adolescence : différences entre filles et garçons. Enfance, 56(4), 405-420.

Smith, N., Lam, D., Bifulco, A., \& Checkley, S. (2002). Childhood Experience of Care and Abuse Questionnaire (CECA.Q): validation of a screening instrument for childhood adversity in clinical populations. Social Psychiatry and Psychiatric Epidemiology, 37(12), 572-579.

Song, L. Y., Singh, J., \& Singer, M. (1994). The Youth Self-Report inventory: A study of its measurements fidelity. Psychological Assessment, 6(3), 236.

Thomaes, S., Bushman, B. J., de Castro, B. O., Cohen, G. L., \& Denissen, J. J. (2009). Reducing narcissistic aggression by buttressing self-esteem: An experimental field study. Psychological Science, 20(12), 1536-1542.

Thomaes, S., Stegge, H., Bushman, B. J., Olthof, T., \& Denissen, J. (2008). Development and validation of the Childhood Narcissism Scale. Journal of personality assessment, 90(4), 382-391.

Tracy, J., L., Cheng, J., T., Robins, R. W. \& Trzesniewski, K., H. (2009). Authentic and hubristic pride: the affective core of self-esteem and narcissism. Self and Identity, 8(2),196-213.

Twenge, J. M., \& Campbell, W. K. (2003). 'Isn’ $t$ it fun to get the respect that we' re going to deserve?' Narcissism, social rejection, and aggression. Personality and Social Psychology Bulletin, 29, 261-272. 
van der Kolk, B. (2016). Commentary: The devastating effects of ignoring child maltreatment in psychiatry--a commentary on Teicher and Samson 2016. Journal of Child Psychology and Psychiatry, 57, 267-270. doi:10.1111/jcpp.12540

Waxman, R., Fenton, M. C., Skodol, A. E., Grant, B. F., \& Hasin, D. (2014). Childhood maltreatment and personality disorders in the USA: specificity of effects and the impact of gender. Personality and Mental Health, 8, 30-41. doi:10.1002/pmh.1239

Wilson, M.S., \& Sibley, C.G. (2011). “Narcissism creep?”: Evidence for age-related differences in narcissism in the New Zealand general population. New Zealand Journal of Psychology, 40(3), 89-95.

Widom, C. S., Czaja, S. J., \& Dutton, M. A. (2008). Childhood victimization and lifetime revictimization. Child abuse \& neglect, 32(8), 785-796.

Wright, A. G. C., Lukowitsky, M. R., Pincus, A. L., \& Conroy, D. E. (2010). The higher order factor structure and gender invariance of the Pathological Narcissism Inventory. Assessment, 17(4), 467-483.

Zanarini, M.C., Williams, A.A., Lewis, R.E., Reich, R.B., Vera, S.C., Marino, M.F., ... Frankenburg, F.R. (1997). Reported pathological childhood experiences associated with the development of borderline personality disorder. American Journal of Psychiatry, 154, 1101-1106.

Zhou, H., Li, Y., Zhang, B., \& Zeng, M. (2012). The relationship between narcissism and friendship qualities in adolescents: Gender as a moderator. Sex roles, 67, 452-462. 


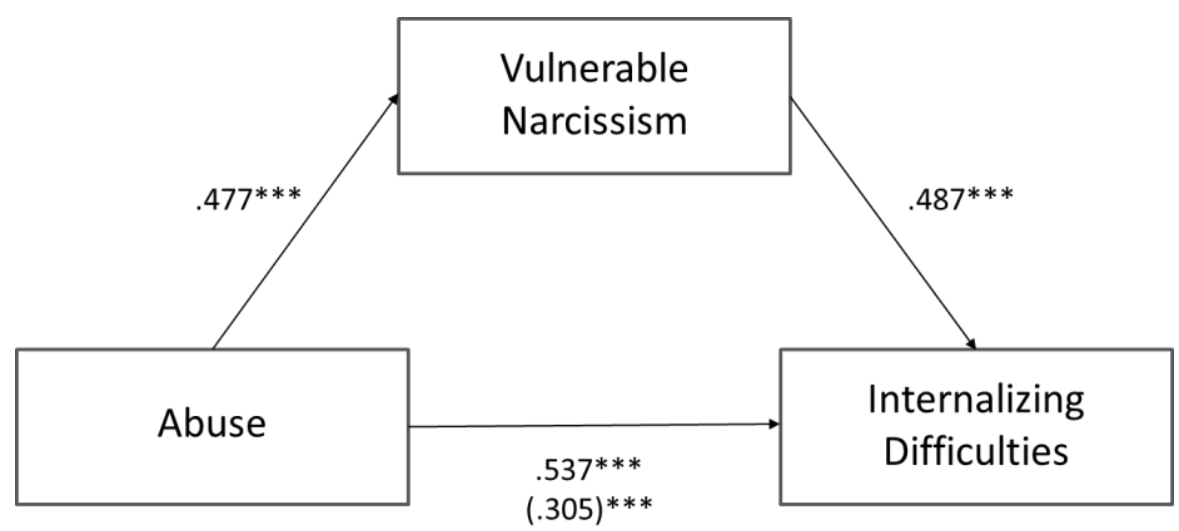

Figure 1. Standardized regression coefficients for the association between internalizing difficulties and abuse partially mediated by vulnerable narcissism in girls.

Note: Standardized regression coefficients are provided with the controlled coefficient in parentheses. $* * * p<.001$. 


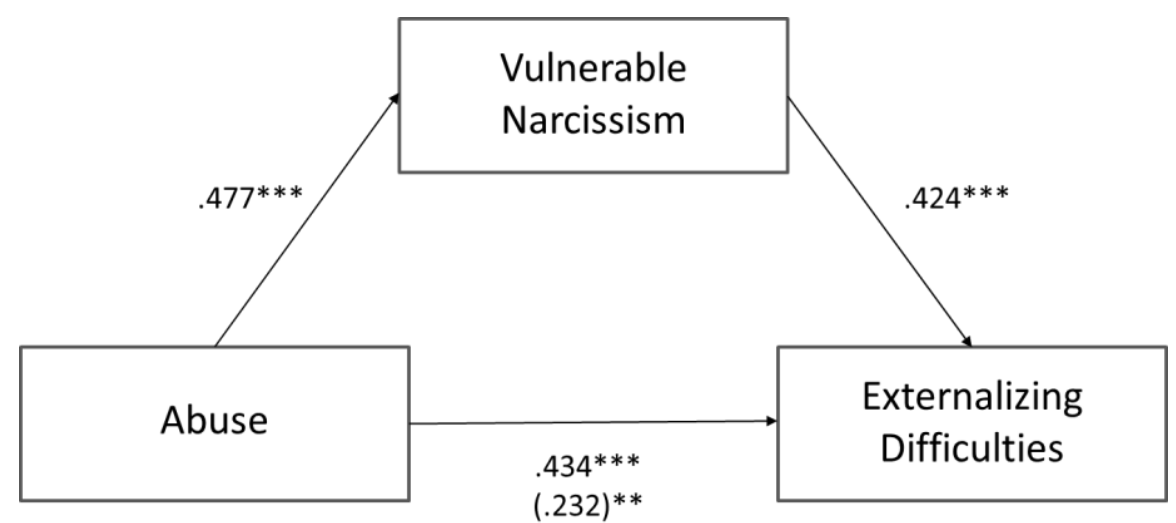

Figure 2. Standardized regression coefficients for the association between externalizing difficulties and abuse partially mediated by vulnerable narcissism in girls.

Note: Standardized regression coefficients are provided with the controlled coefficient in parentheses. $* * * p<.001, * * p<.01$ 


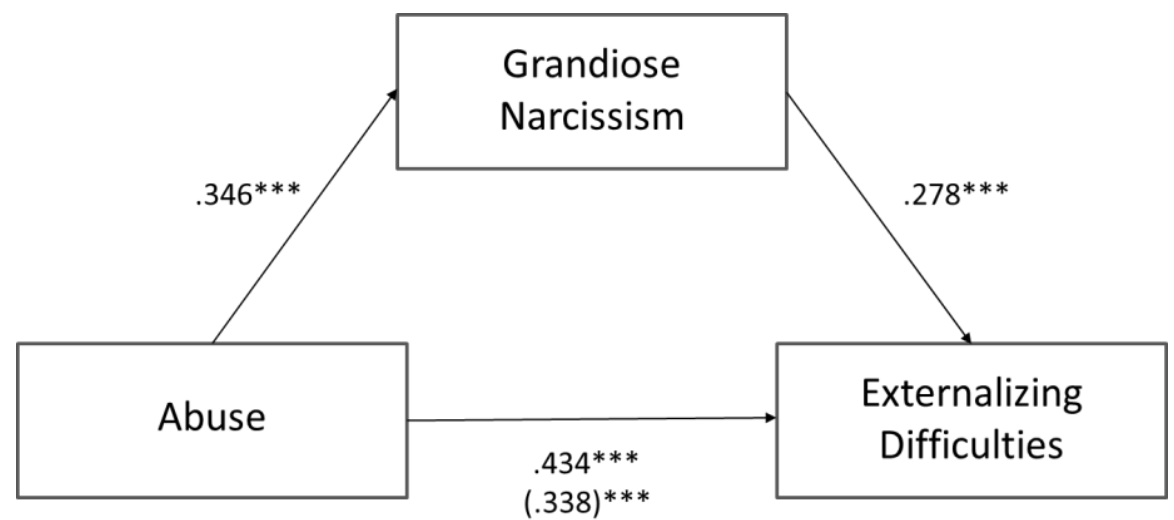

Figure 3. Standardized regression coefficients for the association between externalizing difficulties and abuse partially mediated by grandiose narcissism in girls

Note: Standardized regression coefficients are provided with the controlled coefficient in parentheses. $* * * p<.001$. 
Table 1.

Correlations between age and the facets of the Pathological Narcissism Inventory (PNI) for girls and boys.

\begin{tabular}{|c|c|c|}
\hline & Boys & Girls \\
\hline Facets & \multicolumn{2}{|c|}{ Age } \\
\hline Contingent Self Esteem & $.331 * *$ & -.065 \\
\hline Hiding Self & $.387 * *$ & -.091 \\
\hline Grandiose Fantasy & $.282 * *$ & $-.122 *$ \\
\hline Entitlement Rage & .093 & $-115^{*}$ \\
\hline Devaluation & $.346 * *$ & -.093 \\
\hline Self Sacrificing Self Enhancement & .171 & $-.145 * *$ \\
\hline Eploitation & $.298 * *$ & -.001 \\
\hline Grandiose & $.327 * *$ & $-.116^{*}$ \\
\hline Vulnerable & $.324 * *$ & $-.112 *$ \\
\hline Total & $.389 * *$ & $-.127 *$ \\
\hline
\end{tabular}

Note: $* p<.05 ; * * p<.01$. 
Table 2.

Correlations between pathological narcissism, internalizing and externalizing difficulties, and neglect and abuse.

\begin{tabular}{|c|c|c|c|c|c|}
\hline Total sample & 1 & 2 & 3 & 4 & 5 \\
\hline \multicolumn{6}{|l|}{ 1.PNI vulnerable } \\
\hline 2.PNI grandiose & $.564 * * *$ & & & & \\
\hline 3.PNI total & $.925 * * *$ & $.836 * * *$ & & & \\
\hline 4.Internalizing difficulties & $.610 * * *$ & $.266 * * *$ & $.533 * * *$ & & \\
\hline 5.Externalizing difficulties & $.527 * * *$ & $.369 * * *$ & $.524 * * *$ & $.572 * * *$ & \\
\hline 6.Abuse & $.435 * * *$ & $.266 * * *$ & $.420 * * *$ & $.484 * * *$ & $.425 * * *$ \\
\hline Girls & 1 & 2 & 3 & 4 & 5 \\
\hline \multicolumn{6}{|l|}{ 1.PNI vulnerable } \\
\hline 2.PNI grandiose & $.585 * * *$ & & & & \\
\hline 3.PNI total & $.932 * * *$ & $.840 * * *$ & & & \\
\hline 4.Internalizing difficulties & $.631 * * *$ & $.315 * * *$ & $.571 * * *$ & & \\
\hline 5.Externalizing difficulties & $.544 * * *$ & $.408 * * *$ & $.553 * * *$ & $.588 * * *$ & \\
\hline 6.Abuse & $.487 * * *$ & $.354 * * *$ & $.498 * * *$ & $.527 * * *$ & $.406 * * *$ \\
\hline Boys & 1 & 2 & 3 & 4 & 5 \\
\hline \multicolumn{6}{|l|}{ 1.PNI vulnerable } \\
\hline 2.PNI grandiose & $.627 * * *$ & & & & \\
\hline 3.PNI total & $.932 * * *$ & $.867 * * *$ & & & \\
\hline 4.Internalizing difficulties & $.492 * *$ & .129 & $.378 * *$ & & \\
\hline 5.Externalizing difficulties & $.437 * *$ & .213 & $.381 * *$ & $.474 * *$ & \\
\hline 6.Abuse & .249 & -.128 & .108 & .332 & $.549 * * *$ \\
\hline
\end{tabular}

Note: $* * \mathrm{p}<.01, * * * \mathrm{p}<.001$ 